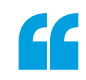

The actuating properties of the fluorenone crystal are very strong

\title{
Robust, reversible and reliable
}

Molecular crystals can experience large changes in shape in response to external stimuli such as heat and light. This type of mechanical response may be harnessed for use in actuation devices in soft robots and artificial muscles. However, for brittle organic crystals, these shape changes can cause the material to break or even explode. Thus, organic molecular crystals that are robust and can reversibly respond to a stimulus, over many cycles without showing signs of wear, are sought after.

Now, writing in Nature Communications, Jialiang $\mathrm{Xu}$, Theo Rasing and colleagues report a fluorenone-derived molecule that dramatically and abruptly changes

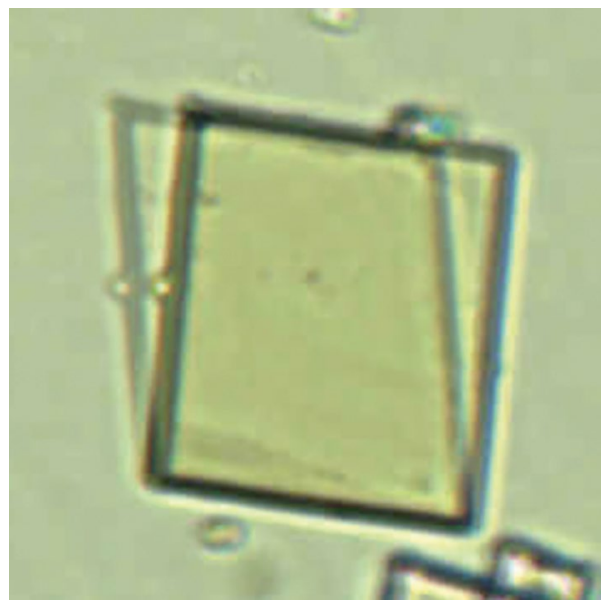

Credit: Reproduced from Duan et al., Nat. Commun. 10, 4573, CC-BY-4.0 https://creativecommons.org/licenses/by/4.0/ shape with temperature, such that if the material is resting on a particular crystal facet, it jumps up a few millimetres. "The actuating properties of the fluorenone crystal are very strong - corresponding to a force about 10,000 times the weight of the crystal." explains Rasing. "Also, the crystal remains intact after hundreds of cycles."

These macroscopic effects are a result of small conformational changes that occur within the crystal structure when it transitions between the $\alpha$-phase and $\beta$-phase at just below $180^{\circ} \mathrm{C}$. "Moreover, the actuation occurs upon both heating and cooling, and every crystal has two different deformation directions," says Rasing.

Below the phase transition temperature, the fluorenone derivative is a parallelogram-shaped, thin crystal (the $\alpha$-phase), whereas above this temperature the crystal becomes more oblique in shape (the $\beta$-phase). Overlapping microscopy images taken before and after the phase transition, show the difference in shape between the two phases (see image).

The researchers use this shear deformation, within the narrow temperature window of the phase transition, to move a micron-sized glass bead resting on a substrate coated with the crystal. From these experiments, the researchers estimate that the output of the kinetic energy upon deformation is 65 pJ. "Also, after applying a large load, the phase boundary becomes incoherent rather than the crystal breaking, which may provide new insights into the molecular movements during the phase transition," says Rasing.

The reversible nature of the molecular actuator is a consequence of the specific rotations that occur between the central fluorenone moiety and the two phenyl rings linked, on either side, by single bonds. To understand the mechanism of the process further, the researchers perform molecular dynamics simulations. "The simulations tell us that the transition proceeds through a very fast nucleation-and-growth mechanism, whereas it is usually assumed to proceed through a more collective mechanism," says Rasing.

In future studies, the researchers envisage using terahertz-visible pump-probe experiments to investigate the microscopic details of the molecular phase transition. "From what we understand of the process, we would then like to design and synthesize molecules where the phase transition in the crystal is closer to room temperature," concludes Rasing. Alison Stoddart

ORIGINAL ARTICLE Duan, Y. et al. Robust thermoelastic microactuator based on an organic molecular crystal. Nat. Commun. 10, 4573 (2019) 